\title{
Further Investigation of Equilibrium Modulus of Diblock Copolymer Micellar Lattice
}

\author{
Hendra TAN and Hiroshi WATANABE ${ }^{\dagger}$ \\ Institute for Chemical Research, Kyoto University, Uji, Kyoto 611-0011, Japan
}

(Received January 15, 2004; Accepted March 10, 2004; Published May 15, 2004)

\begin{abstract}
Polystyrene-polybutadiene (PS-SB) and polystyrene-polyisoprene (PS-PI) diblock copolymers form spherical micelles with PS cores and PB/PI corona in a PB/PI-selective solvent, $n$-tetradecae (C14). At high concentrations, the micelles are further arranged on bcc lattices. Structural factors governing the equilibrium modulus $G_{\mathrm{e}}$ of these PS-PB and PS-PI micellar lattices are discussed in this study. It turned out that $G_{\mathrm{e}}$ is primarily determined by the number density of the corona blocks $v_{\text {corona }}$ and proportional to $v_{\text {corona }}$ while the number density of the bcc lattice cell, $D^{-3}$ with $D$ being the lattice spacing (determined from SAXS), has only secondary effects on $G_{\mathrm{e}}$. The proportionality between $G_{\mathrm{e}}$ and $v_{\text {corona }}$ reflects the thermodynamic origin of the lattice elasticity, the entropic elasticity of the corona $\mathrm{PB} / \mathrm{PI}$ blocks having osmotically correlated conformations. These results suggest a necessity of re-examination of a previous hypothesis that the cell number density primarily determines $G_{\mathrm{e}}$ of bulk copolymer systems forming cubic phases [Kossuth et al., J. Rheol., 43, 167 (1999)]. Furthermore, the $G_{\mathrm{e}} / v_{\text {corona }}$ ratio was found to be moderately different for the PS-PB/C14 and PS-PI/C14 micellar lattices. This difference can be related to a difference in the magnitude of the osmotic correlation for the PB and PI corona blocks due to a slight difference of their solubilities in C14. [DOI 10.1295/polymj.36.430]

KEY WORDS Diblock Copolymer Micellar Lattice / Equilibrium Modulus / Osmotic Requirement / Lattice Stability /
\end{abstract}

Diblock copolymers form micelles with precipitated cores and solvated corona in selective solvents. These micelles form cubic lattices at high concentrations where the corona blocks of neighboring micelles are overlapping each other. ${ }^{1-6}$ This lattice formation results from a compromise of contradicting thermodynamic requirements, an elastic requirement of randomizing corona conformation and an osmotic requirement of reducing the spatial gradient of the corona concentration. ${ }^{1,4-6}$ Under these requirements, the corona blocks of neighboring micelles are forced to have mutually correlated conformations. The micelles are arranged on cubic lattices so as to satisfy the thermodynamic requirements and minimize this correlation.

The micellar lattice is the thermodynamically stable structure and exhibits equilibrium elasticity. ${ }^{1-6}$ For polystyrene-polybutadiene (PS-PB) copolymers in a PB-selective solvent, $n$-tetradecane (C14), the equilibrium modulus $G_{\mathrm{e}}$ was found to be proportional to the number density $v_{\text {corona }}$ of the corona PB blocks and the proportionality constant $\left(G_{\mathrm{e}} / v_{\text {corona }}\right.$ ratio $)$ was smaller than the constant $k_{\mathrm{B}} T$ expected for the simplest case of the entropic elasticity of independent corona blocks $\left(k_{\mathrm{B}}=\right.$ Boltzmann constant and $T=\mathrm{ab}-$ solute temperature). ${ }^{4-6}$ This proportionality reflects the thermodynamic origin of the lattice. Namely, the osmotically correlated corona blocks sustain the lat- tice and thus their number density is the primary factor determining $G_{\mathrm{e}}$. At the same time, respective corona blocks do not behave as an independent entropic unit because of their conformational correlation. ${ }^{7}$ This correlation leads the $G_{\mathrm{e}} / v_{\text {corona }}$ ratio smaller than $k_{\mathrm{B}} T$. Indeed, an experiment demonstrated that the $G_{\mathrm{e}}$ value increases moderately when the solvent quality toward the corona block is mildly reduced to partially screen this correlation. ${ }^{8}$

Thus, the equilibrium elasticity of the micellar lattice in selective solvents is well related to the thermodynamic (osmotic) feature of the corona blocks. However, Kossuth et al. ${ }^{9}$ recently reported that $G_{\mathrm{e}}$ of microphase-separated bulk block copolymers forming cubic lattices is primarily determined by the lattice spacing $D$ and proportional to the number density of the lattice cell, $D^{-3}$. Their result, if applicable to all cubic lattices, gives us an interpretation that the lattice cell itself is the fundamental entropic unit sustaining the elasticity and the (correlated) corona blocks do not behave as this unit. This interpretation is contradicting to the thermodynamic feature of the micellar lattice (corona-sustained elasticity) confirmed from several experiments. ${ }^{2-7}$

For this problem, we have examined a relationship between $G_{\mathrm{e}}$ and $D^{-3}$ for a series of PS-PB and polystyrene-polyisoprene (PS-PI) micellar lattices in C14. It turned out that the $G_{\mathrm{e}}$ data of respective series scale

${ }^{\dagger}$ To whom correspondence should be addressed (E-mail: hiroshi@scl.kyoto-u.ac.jp). 
with $v_{\text {corona }}$ but not with $D^{-3}$ thereby lending support to the molecular picture of the corona-sustained elasticity. (This picture appeared to apply also to bulk copolymers.) Furthermore, the $G_{\mathrm{e}} / v_{\text {corona }}$ ratio was found to be moderately different for the PS-PB and PS-PI micellar lattices, suggesting a difference in the magnitude of the osmotic correlation for the $\mathrm{PB}$ and PI corona blocks due to a slight difference of the solubilities of PB and PI in C14. Details of these results are presented in this paper.

\section{EXPERIMENTAL}

\section{Materials}

PS-PI and PS-PB diblock copolymers were anionically polymerized with sec-butyllithium in benzene. PI and/or PB blocks were polymerized first, and their living anions were split into several batches. Then, the PS blocks of various lengths were copolymerized in respective batches. For convenience of the molecular characterization, the precursor PI/PB blocks were also recovered.

These copolymer samples were characterized with GPC (CO-8020 and DP-8020, Tosoh Corporation) equipped with refractive index (RI) and ultraviolet absorption (UV) monitors (LS-8000 and UV-8020, Tosoh Corporation) connected in series. The eluent was THF. The PI/PB molecular weights were determined for the precursors through elution volume calibration utilizing previously synthesized/characterized monodisperse linear PI and PB samples ${ }^{10-12}$ as the elution standards. The PS molecular weight was determined from the RI and UV signal intensities of the copolymers.

The systems subjected to viscoelastic and small-angle X-Ray scattering (SAXS) measurements were the copolymer solutions in $n$-tetradecane (C14; Wako Pure Chemical Industries, Ltd.). These solutions were prepared by firstly dissolving prescribed masses of the copolymer and $\mathrm{C} 14$ in excess methylene chloride and then allowing methylene chloride to thoroughly evaporate. C14 is a good solvent for PI and PB blocks and a non-solvent for the PS blocks. Thus, the copolymers formed micelles with precipitated PS cores and solvated PB/PI corona in C14, as confirmed from SAXS measurements.

\section{Measurements}

For the PS-PI/C14 and PS-PB/C14 micellar solutions of various concentrations, linear viscoelastic measurements were carried out at $25^{\circ} \mathrm{C}$ with a laboratory rheometer (ARES; Rheometrics F. E. Ltd). These solutions were charged in the rheometer and disordered at $70-90{ }^{\circ} \mathrm{C}$ for $15 \mathrm{~min}$ and then quiescently ordered (without being deformed) by cooling to $25^{\circ} \mathrm{C}$.
This process enabled equilibration of the micellar lattice by erasing undesirable shear history during the sample loading. After annealing at $25^{\circ} \mathrm{C}$ for $12 \mathrm{~h}$, the storage and loss moduli, $G^{\prime}$ and $G^{\prime \prime}$, were measured under sufficiently small strain $(\leq 0.01)$.

For the micellar solutions, small-angle X-Ray scattering (SAXS) measurements were also conducted at $25^{\circ} \mathrm{C}$ with a laboratory goniometer (RINT-2000, Rigaku Corp.) equipped in Professor Kanaya's laboratory in Institute for Chemical Research, Kyoto University. $\mathrm{Cu}-\mathrm{K} \alpha$ line of the wavelength $\lambda=0.154 \mathrm{~nm}$ was used. The samples were charged in a cell having a mica window. The SAXS intensity $I(q)$ was measured as a function of the magnitude of the scattering vector $q=4 \pi \sin \theta / \lambda$ ( $\theta=$ scattering angle). No desmearing correction was made for the $I(q)$ data.

\section{RESULTS AND DISCUSSION}

\section{Viscoelastic and SAXS Data}

Linear viscoelastic and SAXS measurements were conducted for the PS-PI/C14 and PS-PB/C14 systems of various concentrations at $25^{\circ} \mathrm{C}$. Representative results obtained for the $31.0 \mathrm{wt} \%$ PS-PI 11-85/ C14 system are summarized in Figures 1 and 2: Figure 1 shows angular frequency $(\omega)$ dependence

Table I. Characteristics of Diblock Copolymer Samples

\begin{tabular}{|c|c|c|c|}
\hline $\operatorname{Code}^{\mathrm{a}}$ & $10^{-3} M_{\mathrm{S}}$ & $10^{-3} M_{\mathrm{D}}^{\mathrm{b}}$ & $M_{\mathrm{w}} / M_{\mathrm{n}}$ \\
\hline PS-PI $11-85$ & 10.5 & 85.0 & 1.03 \\
\hline PS-PI 19-85 & 18.7 & 85.0 & 1.03 \\
\hline PS-PI 42-85 & 41.9 & 85.0 & 1.03 \\
\hline PS-PB 13-93 & 12.7 & 92.8 & 1.03 \\
\hline PS-PB 23-93 & 23.2 & 92.8 & 1.03 \\
\hline
\end{tabular}

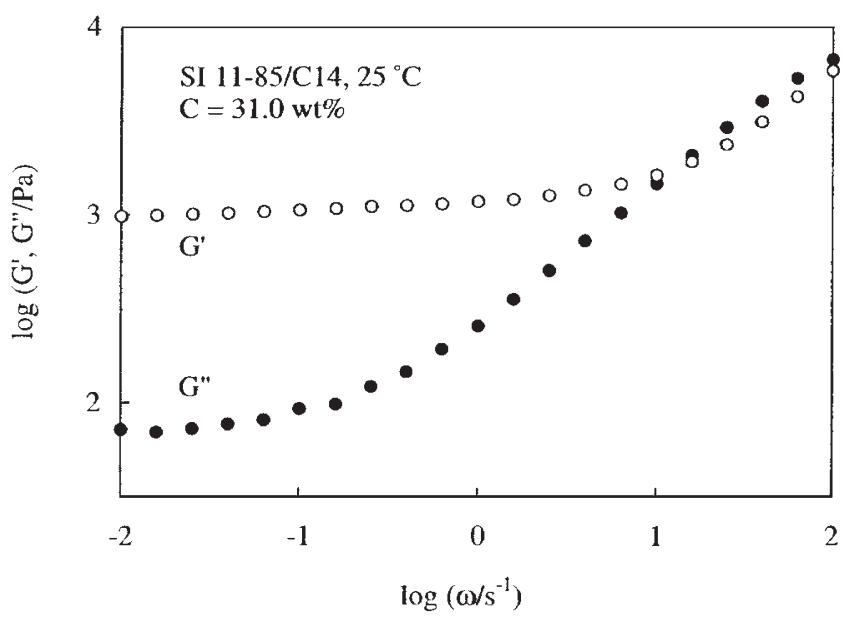

Figure 1. Linear viscoelastic behavior of the $31.0 \mathrm{wt} \%$ PS-PI $11-85 / \mathrm{C} 14$ micellar lattice system at $25^{\circ} \mathrm{C}$. 


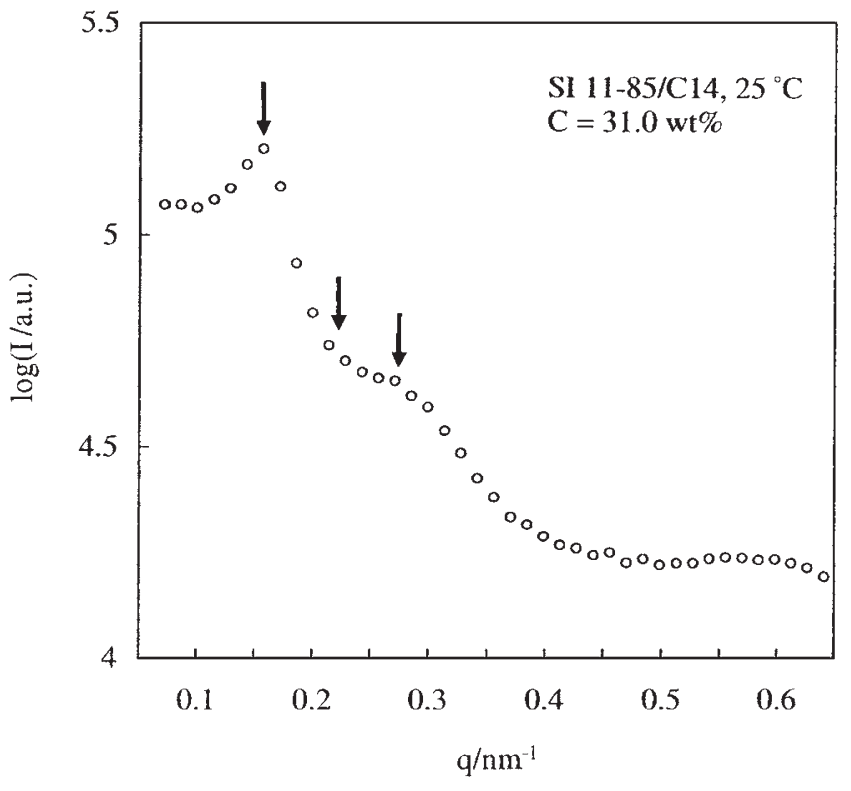

Figure 2. SAXS profile of the $31.0 \mathrm{wt} \%$ PS-PI $11-85 / \mathrm{C} 14$ micellar lattice system at $25^{\circ} \mathrm{C}$.

of the storage and loss moduli $G^{\prime}$ and $G^{\prime \prime}$, and Figure 2 shows the $q$ dependence of the SAXS intensity $I(q)$.

Judging from a small volume fraction $(=0.036)$ of the PS block in the PS-PI 11-85/C14 system, we expect that the PS-PI 11-85 chains form spherical micelles with precipitated PS cores and solvated PI corona. This expectation is confirmed in Figure 2 where the scattering peaks in a range of $q<0.4 \mathrm{~nm}^{-1}$ are assigned to inter-domain interference in the micellar lattice. In addition, the scattering maximum at $q \cong$ $0.6 \mathrm{mn}^{-1}$ is attributable to the intra-domain scattering from the PS cores of the radius $r_{\mathrm{S}}=5.765 / q=9 \pm$ $0.5 \mathrm{~nm}$. This $r_{\mathrm{S}}$ value is a little larger than the PS domain radius expected in bulk systems $(\cong 8 \mathrm{~nm}),{ }^{6}$ suggesting that the PS block of $M_{\mathrm{S}}=10.5 \times 10^{3}$ is moderately swollen and plasticized with $\mathrm{C} 14$. Indeed, a thermal analysis for a little shorter PS block $\left(M_{\mathrm{S}}=\right.$ $7.2 \times 10^{3}$ ) revealed the swelling of this block with C14 that decreased the glass transition temperature $T_{\mathrm{g}}$ to $30^{\circ} \mathrm{C} .{ }^{13}$ At the same time, it should be emphasized that our PS block is longer and should have $T_{\mathrm{g}}>30^{\circ} \mathrm{C}$ to behave as a rigid (glassy) anchor for the PI corona at our experimental temperature, $25^{\circ} \mathrm{C}$. This was the case for all PS-PI and PS-PB copolymers examined in this study.

The arrows in Figure 2 indicate the relative location of the inter-domain scattering peaks expected for a body-centered cubic (bcc) lattice of the spherical micelles, $q_{1}: q_{2}: q_{3}=1: \sqrt{2}: \sqrt{3}$. Although the second-order peak is not well resolved, the relative location of the first- and third-order peaks coincides with that for the bcc lattice to confirm the bcc lattice formation of the spherical PS-PI 11-85 micelles. This lattice formation was confirmed for all PS-PI/C14 and PS-
$\mathrm{PB} / \mathrm{C} 14$ systems examined.

In Figure 1, we note that $G^{\prime}$ and $G^{\prime \prime}$ of $31.0 \mathrm{wt} \%$ PS-PI 11-85/C14 system are close to each other in magnitude and exhibit considerable $\omega$ dependence in a range of $\omega>1 \mathrm{~s}^{-1}$. This behavior is indicative of a fast relaxation process (attributable to motion of the PI blocks). However, at $\omega<1 \mathrm{~s}^{-1}, G^{\prime}$ is quite insensitive to $\omega$ and $G^{\prime \prime}$ is much smaller than $G^{\prime}$. This low- $\omega$ behavior clearly demonstrates the equilibrium elasticity of the micellar lattice formed in the system. Similar behavior was observed for all systems examined.

\section{Structural Factors Determining Equilibrium Modulus}

For all PS-PI/C14 and PS-PB systems, the elastic behavior was observed at low $\omega$ and the equilibrium modulus $G_{\mathrm{e}}$ was evaluated as the $G^{\prime}$ at the lowest $\omega$ examined $\left(=0.01 \mathrm{~s}^{-1}\right)$. Figures 3 shows double-logarithmic plots of the normalized modulus $G_{\mathrm{e}} / k_{\mathrm{B}} T$ against the number density of the corona blocks $v_{\text {corona }}$ (calculated from the copolymer concentration and molecular weight). The data obtained in this study are shown with large unfilled symbols. For comparison, the plots are also shown for previously examined PS-PB/C14 1,2,7,14 and PS-PI/C14 ${ }^{13,15,16}$ micellar lattice systems (small unfilled symbols) and for bulk diblock copolymers forming cubic lattices reported by Kossuth et al. ${ }^{9}$ (filled symbols), poly(ethylene propylene)-poly(ethyl ethylene) (PEP-PEE), polyethylenepoly(ethyl ethylene) (PE-PEE), PI-PS, and poly(ethylene oxide)-poly(ethyl ethylene) (PEO-PEE).

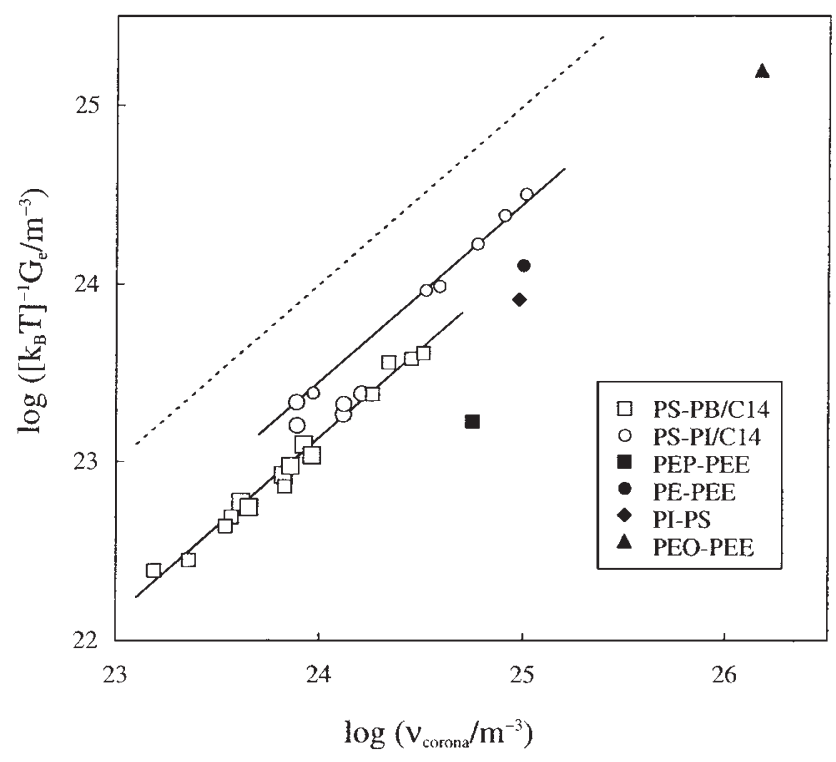

Figure 3. Plots of the normalized equilibrium modulus $G_{\mathrm{e}} / k_{\mathrm{B}} T$ of various copolymer lattice systems against the corona number density $v_{\text {corona. }}$. The dotted line indicates a relationship for the simplest case of the entropic elasticity of independent corona blocks, $G_{\mathrm{e}}{ }^{\circ} / k_{\mathrm{B}} T=v_{\text {corona }}$. 


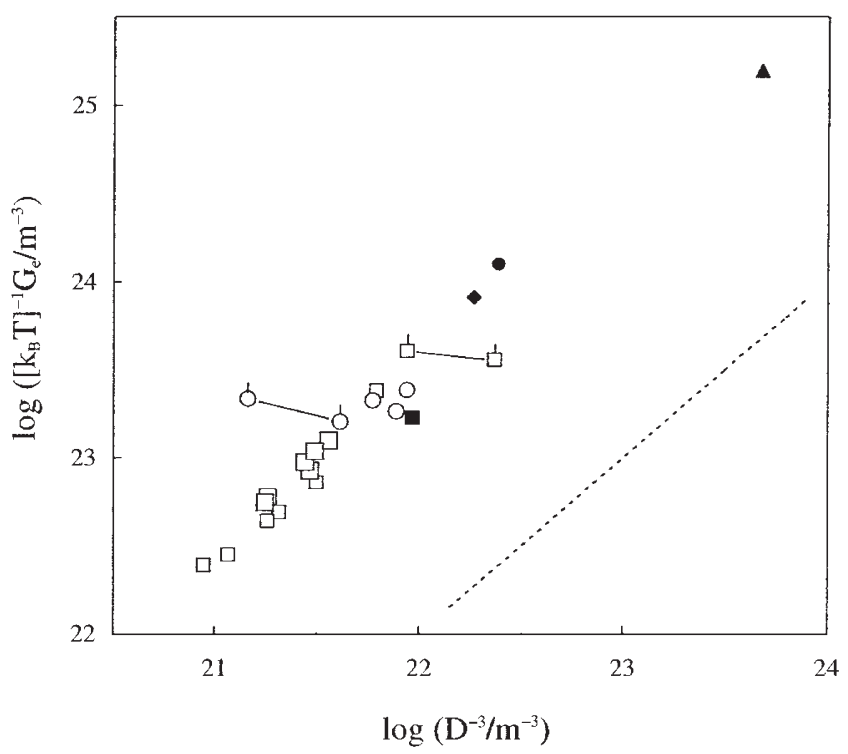

Figure 4. Plots of the normalized equilibrium modulus $G_{\mathrm{e}} / k_{\mathrm{B}} T$ of various copolymer lattice systems against the number density $D^{-3}$ of the bcc lattice cell. The dotted line indicates a relationship, $G_{\mathrm{e}} / k_{\mathrm{B}} T=D^{-3}$. This relationship is to be satisfied if each cell behaves as the independent entropic unit sustaining the lattice elasticity.

The corona concentration is in a range of $0.08 \leq$ $c_{\text {corona }} / \mathrm{g} \mathrm{cm}^{-3} \leq 0.25$ for all PS-PB $/ \mathrm{C} 14$ and PS$\mathrm{PI} / \mathrm{C} 14$ lattice systems examined here.

The spacing $D$ of the bcc lattice formed in the above systems is evaluated from the magnitude of the scattering vector at the first-order peak, $q_{1}$, as

$$
D=\sqrt{\frac{3}{2}} \frac{2 \pi}{q_{1}}
$$

For the lattice systems for which both of the $G_{\mathrm{e}}$ and $D$ data are available, Figure 4 shows the plots of $G_{\mathrm{e}} / k_{\mathrm{B}} T$ against the number density of the bcc lattice cell, $D^{-3}$. The symbols are the same as in Figure 3. [Kossuth et al. ${ }^{9}$ made plots of $G_{\mathrm{e}} / R T$ ( $R=$ gas constant) against $2 \pi / q_{1}$. The format of the plots in Figure 4 is equivalent to their format.]

In Figure 3, we first note that the $G_{\mathrm{e}} / k_{\mathrm{B}} T$ data of all systems are smaller than the normalized modulus expected for the simplest case of the entropic elasticity of independent corona blocks, $G_{\mathrm{e}}{ }^{\circ} / k_{\mathrm{B}} T=v_{\text {corona }}$ (shown with the dotted line). This result is naturally related to the thermodynamic origin of the lattice: The corona blocks are conformationally correlated under the osmotic requirement and thus the effective number density of the independent entropic unit (a set of correlated corona blocks) is smaller than $v_{\text {corona }}$ thereby giving $G_{\mathrm{e}} / k_{\mathrm{B}} T<v_{\text {corona }}$.

This statement can be re-cast in the thermodynamic expression of $G_{\mathrm{e}}$ of micellar lattices derived by Hashimoto et al. ${ }^{14}$

$$
\frac{G_{\mathrm{e}}}{k_{\mathrm{B}} T}=\frac{v_{\text {corona }}}{\beta}
$$

with

$$
\frac{1}{\beta}=\frac{1}{\gamma^{2}} \ln \left(\frac{P_{0}}{P}\right) \text { for small } \gamma
$$

where $P_{0}$ is the probability density of the osmotically constrained corona blocks in the undeformed state and $P$ is the probability density of these blocks under a small strain $\gamma$. (Since the $\ln \left(P_{0} / P\right)$ term does change with the direction of shear, this term should be proportional to $\gamma^{2}$ for small $\gamma$.) The parameter $\beta$ appearing in Eq 2 is equivalent to the number of the corona blocks per independent entropic unit explained above. This $\beta$ would increase from unity (for the case of independent Gaussian blocks) with increasing magnitude of the osmotic constraint. However, for respective series of the PS-PI/C14 and PS-PB/C14 micellar lattices in the rather limited range of $0.08 \leq c_{\text {corona }} / \mathrm{g} \mathrm{cm}^{-3} \leq$ $0.25, \beta$ appears to be roughly constant to give the proportionality between $G_{\mathrm{e}} / k_{\mathrm{B}} T$ and $v_{\text {corona }}$ within experimental scatter (Figure 3).

Nevertheless, $\beta$ would be determined by the osmotic compressibility for the solvated corona blocks ${ }^{8}$ and may have different values in the systems comprising of chemically different blocks/solvents. Concerning this difference, we note that the $G_{\mathrm{e}} / k_{\mathrm{B}} T$ value for a given $v_{\text {corona }}$ value is somewhat larger for the PSPI/C14 systems than for the PS-PB/C14 systems; $c f$. Figure 3. This result reminds us a previous observation $^{8}$ that $G_{\mathrm{e}}$ of PS-PI lattices moderately increases (by a factor up to 60\%) on replacement of a small fraction (7\%) of the solvent $\mathrm{C} 14$ by a non-solvent, diethyl phthalate: This replacement resulted in a slight reduction of the solubility for the PI corona, as characterized by a small decrease $(6 \%)$ of the intrinsic viscosity of a high- $M$ homo-PI $\left(M=180 \times 10^{3}\right)$ on the replacement. Judging from this sensitivity of $G_{\mathrm{e}}$ to the osmotic environment for the corona (determined by the solubility), we speculate that the moderate difference in the $G_{\mathrm{e}} / k_{\mathrm{B}} T$ values for the PS-PI/C14 and PS-PB/C14 systems (Figure 3) may reflect a slight difference of the solubilities of PI and PB blocks in C14. (As judged from a difference of the Mark-Houwink-Sakurada exponents $\alpha$ in hexane/heptane chemically similar to $\mathrm{C} 14, \alpha=0.58$ for $\mathrm{PI} /$ hexane solutions $^{17}$ and $\alpha=0.64$ for $\mathrm{PB} /$ heptane $^{18}$ solutions (both values obtained from five fractions), C14 appears to be slightly poorer for PI than for PB thereby giving somewhat larger $G_{\mathrm{e}}$ for PS-PI.) A further study is desirable for this delicate solvent effect.

Now, we turn our attention to the relationship between $G_{\mathrm{e}}$ and $D^{-3}$. Since $D$ for a given copolymer decreases with an increase of the copolymer concentra- 
tion, a general trend in the $G_{\mathrm{e}} / k_{\mathrm{B}} T$ vs. $D^{-3}$ plots (Figure 4) is similar to that in the $G_{\mathrm{e}} / k_{\mathrm{B}} T v s . v_{\text {corona }}$ plots (Figure 3 ). However, a considerably large scatter is observed in the $G_{\mathrm{e}} / k_{\mathrm{B}} T$ vs. $D^{-3}$ plots for respective series of the PS-PI/C14 and PS-PB/C14 systems; see the unfilled circles, for example. This scatter suggests that the bcc lattice cell is not the independent entropic unit sustaining the lattice elasticity. This point can be most clearly noted for the two pip-circles indicating the $G_{\mathrm{e}}$ data for the $16.6 \mathrm{wt} \%$ PS-PI $19-85 / \mathrm{C} 14$ and $20.0 \mathrm{wt} \%$ PS-PI 42-85/C14 systems having quite different $D$ values but identical $v_{\text {corona }}$ value $(=7.8 \times$ $10^{23} \mathrm{~m}^{-3}$ ): $G_{\mathrm{e}}$ hardly changes with $D^{-3}$ if $v_{\text {corona }}$ is kept constant. Similar behavior is noted for the two pip-squares showing $G_{\mathrm{e}}$ of previously examined PS$\mathrm{PB} / \mathrm{C} 14$ systems having quite different $D^{-3}$ values and fairly close $v_{\text {corona }}$ values $\left(=2.2 \times 10^{24}\right.$ and $3.2 \times 10^{24} \mathrm{~m}^{-3}$ ).

If the bcc lattice cell is the independent entropic unit, each cell should sustain the modulus of the order of $k_{\mathrm{B}} T$ and thus $G_{\mathrm{e}} / k_{\mathrm{B}} T$ should be close to the number density of the cell, $D^{-3}$. However, Figure 4 demonstrates that the $G_{\mathrm{e}} / k_{\mathrm{B}} T$ data of all systems are significantly larger (by a factor $>30$ ) than the modulus expected for this case, $G_{\mathrm{e}} / k_{\mathrm{B}} T=D^{-3}$ (dotted line). This fact means that each cell contains many (more than 30) entropic units. This is the case not only for the solvated PS-PB and PS-PI micellar lattices but also for the bulk copolymers examined by Kossuth et al. ${ }^{9}$

The above results allow us to conclude, from both qualitative and quantitative points of view, that the bcc lattice cell is not the independent entropic unit sustaining the lattice elasticity. The cell contains many (more than 30) entropic units. Thus, a set of the conformationally correlated $\beta$ corona blocks should behave as this unit, as concluded from the results shown in Figure 3.

Finally, we should point out that the delicate solvent effect on the number $\beta$ (seen for the PS-PI/ C14 and PS-PB/14 systems) has not been described quantitatively, although the role of the solubility can be explained qualitatively. This description requires us to formulate the probability densities $P$ and $P_{0}$ (Eq 2) as functions of the osmotic compressibility and other thermodynamic parameters. This formulation is considered to be an interesting subject of future work.

\section{CONCLUSIONS}

For the PS-PB/C14 and PS-PI/C14 micellar lattices as well as the lattices in bulk diblock copolymers, the equilibrium modulus $G_{\mathrm{e}}$ was found to be primarily determined by the number density of the corona blocks $v_{\text {corona }}$. For respective series of the micellar lat- tice systems, $G_{\mathrm{e}}$ was proportional to $v_{\text {corona }}$ but smaller, in magnitude, than that expected for the simplest case of the entropic elasticity of the corona blocks, $G_{\mathrm{e}}{ }^{\circ}=v_{\text {corona }} k_{\mathrm{B}} T$. In contrast, no proportionality was found between $G_{\mathrm{e}}{ }^{\circ}$ and the number density of the bcc lattice cell, suggesting that the cell size has only secondary effects on $G_{\mathrm{e}}$. These results indicate that a set of osmotically correlated corona blocks behaves as the independent entropic unit sustaining the lattice elasticity and each cell contains many entropic units. This conclusion holds not only for the solvated micellar lattices but also for the lattices in bulk copolymer systems.

Acknowledgment. The authors cordially thank Professor T. Kanaya, Professor K. Nishida, and Mr. T. Konishi at Instutute for Chemical Research, Kyoto University, for their help in SAXS measurements. H.T. gratefully acknowledges the Jinnai scholarship provided from Association of International Education, Japan.

\section{REFERENCES}

1. H. Watanabe, T. Kotaka, T. Hashimoto, M. Shibayama, and H. Kawai, J. Rheol., 26, 153 (1982).

2. H. Watanabe and T. Kotaka, Polym. J., 14, 739 (1982).

3. H. Watanabe and T. Kotaka, J. Rheol., 27, 223 (1983).

4. H. Watanabe, Nihon Reoroji Gakkaishi (J. Soc. Rheol. Japan), 22, 193 (1994).

5. H. Watanabe, Acta Polym., 48, 215 (1997).

6. H. Watanabe, Kobunshi Ronbunshu, 58, 135 (2001).

7. H. Watanabe, T. Kanaya, and Y. Takahashi, Macromolecules, 34, 662 (2001).

8. H. Tan and H. Watanabe, Polym. J., 34, 775 (2002).

9. M. B. Kossuth, D. C. Morse, and F. S. Bates, J. Rheol., 43, 167 (1999).

10. Y. Matsumiya, H. Watanabe, and K. Osaki, Macromolecules, 33, 499 (2000).

11. H. Watanabe, Y. Matsumiya, and T. Inoue, Macromolecules, 35, 2339 (2002).

12. H. Watanabe, O. Urakawa, H. Yamada, and M. L. Yao, Macromolecules, 29, 755 (1996).

13. H. Watanabe, T. Sato, K. Osaki, M. L. Yao, and A. Yamagishi, Macromolecules, 30, 5877 (1997).

14. T. Hashimoto, M. Shibayama, H. Kawai, H. Watanabe, and T. Kotaka, Macromolecules, 16, 361 (1983).

15. H. Watanabe, T. Sato, and K. Osaki, Macromolecules, 29 , 3890 (1996).

16. H. Watanabe, T. Sato, and K. Osaki, Macromolecules, 33, 2545 (2000).

17. M. Kurata and Y. Tsunashima, "Viscosity-Molecular Weight Relationships and Unperturbed Dimensions of Linear Chain Molecules," in Polymer Handbook, J. Brandrup and E. H. Immergut, Ed., Wiley, New York, N.Y., 1989.

18. O. Urakawa, K. Adachi, and T. Kotaka, Macromolecules, 26, 2036 (1993). 